\title{
Improving Efficiency of CBIR by using Color, Texture and Fusion Features with Bit Pattern
}

\author{
Ramandeep Kaur \\ Department of Computer Science \& Engineering \\ Rajasthan College of Engineering for Women \\ Bhankrota, Ajmer Road, Jaipur - 302026
}

\author{
Vineet Khanna \\ Assistant Professor, Department of Computer \\ Science \& Engineering \\ Rajasthan College of Engineering for Women \\ Bhankrota, Ajmer Road, Jaipur - 302026
}

\begin{abstract}
Digital graphic offers many advantages for processing and distributing image and other types of understanding. A single color image have more information of the different situations and object scenario and each color and texture have own characteristics. Geometrical and color information extraction are the key issue. CBIR finds and show images alike to one given as query image and another are similar images. In the earlier work, most of the researchers used low level features but there are some challenges like distortion of image, color imbalance, semantic gap between low level features etc. The challenges, be that as it may, is in planning a system with the capacity to retrieve best possible matches when there is an occurrence of all kind of images as query without using some other relevant information, for example, metadata, keywords and labels etc, and using only content of the images. The system proposed in this approach aim to retrieve the most relevant images using color, edge histogram descriptor with bitmap by using fusion based feature. Applications of the proposed system are climate forecast, crime prevention, military imaginary etc.
\end{abstract}

\section{Keywords}

EHD, color histogram, Fusion, Color sketch CBIR.

\section{INTRODUCTION}

Digital imaging has played an important role in our life. With the beginning of the twenty century, imaging has grown with an unparalleled way in all our walks of life. Presently, images play a significant role in numerous fields, for example, medication, news coverage, instruction and diversion. By the revolution of PCs, a wide scope of methods helps us for image capturing, processing, storage, and transmitting.

The development of the World-Wide Web enables users to get to information from wherever and gives the exploitation of digital images in any fields [1]. In 1993 the main first internet browser, mosaic, was backslid, and the development rate of the web as yet developing at around $100 \%$ every year.

Query by content: One image is worth ten thousand words really manufactured advertising executive in California in 1927.

There are essentially three different ways of retrieving recently stored mixed media information [2].

Free browsing: Clients peruse through an image collection, video, and sound files, and stop when they locate the desired information.

Text based: Text and literary data is included either manually or using programmed instruments to the varying media documents amid the indexing stage [3].

Content based: client search through the sight and sound archive giving data about the real content of the image, sound, and video clip.

The first two methods have some drawbacks so with the end goal to conquer the wasteful aspects and constraints of text based image retrieval of recently explained visual information, many researchers began to explore conceivable methods for retrieving visual data dependent on its content [4].CBIRSs can be divided into two types: Narrow and Broad Domain Applications.

Table 1. Difference between NDA and BDA

\begin{tabular}{|c|c|c|c|}
\hline $\begin{array}{l}\text { Sr. } \\
\text { No. }\end{array}$ & Description & $\begin{array}{l}\text { NDA(Narrow } \\
\text { domain } \\
\text { Application) }\end{array}$ & $\begin{array}{l}\text { BDA(Broad domain } \\
\text { Application) }\end{array}$ \\
\hline 1 & $\begin{array}{c}\text { Source of } \\
\text { knowledge }\end{array}$ & Target specific & Tackle generic \\
\hline 2 & Variance & Small & High \\
\hline 3 & Semantics & Homogeneous & Heterogeneous \\
\hline 4 & Interactivity & Limited & More \\
\hline 5 & $\begin{array}{c}\text { Content } \\
\text { description }\end{array}$ & More objective & More subjective \\
\hline 6 & $\begin{array}{c}\text { Scenes and } \\
\text { sensor control }\end{array}$ & $\begin{array}{l}\text { May have } \\
\text { control }\end{array}$ & Not have control \\
\hline 7 & Techniques & $\begin{array}{l}\text { Used in object } \\
\text { recognition most } \\
\text { of the times }\end{array}$ & $\begin{array}{l}\text { Used in information } \\
\text { retrieval techniques } \\
\text { most of the times }\end{array}$ \\
\hline 8 & Range & Medium size & $\begin{array}{l}\text { Large to extremely } \\
\text { large size }\end{array}$ \\
\hline 9 & Tools & $\begin{array}{c}\text { Consider } \\
\text { specific in } \\
\text { variances with } \\
\text { model driven } \\
\text { tool }\end{array}$ & $\begin{array}{l}\text { Consider general } \\
\text { invariants with } \\
\text { perceptual /cultural }\end{array}$ \\
\hline 10 & Examples & $\begin{array}{c}\text { MIR, Finger } \\
\text { print retrieval, } \\
\text { satellite images } \\
\text { retrieval }\end{array}$ & $\begin{array}{l}\text { Photo collection and } \\
\text { internet content }\end{array}$ \\
\hline
\end{tabular}




\section{MOTIVATION AND OBJECTIVE}

From a computational point of view, a typical CBIR system views the query image and images in the database (target images) as an accumulation of features, and the retrieval is performed dependent on the significance between the query image and any target images as per their similarity. A portion of the features can be visual features (color, texture, shape) and some can be the human description of the image like impressions and emotions. The second issue is the computational steps to extract features of an image and to manage extensive image databases. Keeping in mind that substantial image databases are used for testing and retrieving the work plans to give a proficient content based image retrieval system. It is expected to overcome the problem of image feature selection to represent the image and to reduce the computation load.Major challenges in such systems are the aggregation of features extracted from multiple images and the computation of similarity between the input query image set and the database images. Considering the abovementioned issues, the main objective of this proposed work is to develop methodologies to improve the retrieval efficiency of broad domain CBIR systems.

Organization: The paper is organized into the following sections. Section II deals with the literature review of few related important papers work in utilized for the dissertation work. In Section IIIproposed methodology. In section IV specified a problem including all the solution and implementation results contains all the evaluation regarding proposed work it deals with MATLAB GUI block and guide. In last section conclusions and future work after applying the proposed approach on the images of the existing work as well as on new images.

\section{LITRATURE REVIEW}

This chapter reviews current literature concerning on CBIR. BMI launched the primary system for CBIR [1] and later, extraordinary image retrieval methods were it dependent on spatial design, texture, color shades, and shape $[1,5]$. CBIR has attracted scientists from many research fields, including Computer vision, man-made consciousness, human factors, and machine learning.

Flickner et al. proposed IBM's QBIC system which is a standout amongst the most outstanding CBIR systems and has a long history. Another quite recent product is Google Image search, which is a feature in the Google search engine "Search by Image". It is optimized to work well for content that is reasonably well depicted on the web, and thus, will in all likelihood give more applicable outcomes for famous landmark or paintings works of art than for more close to home images.

Pushpalatha S. Nikkam et.al. [6] There is a strategy to found a desired image from a considerable database. A layout for shape based various leveled feature matching technique for CBIR system. Shape based global feature formats. The method depends upon hierarchal information decomposition executed to distinguish boundary, introductions and dataset images shape.

Ekta Gupta et.al.[7]Present that CBIR uses visual image content for instance global color features, shape include, texture feature, and nearby features spatial area present to demonstrate and image data. CBIR method joins global and neighborhood features. In this paper SVM classifier used [4].

Kamlesh Kumar et.al.[8] Present CBIR system which has extra consideration from its generic to specific use. CBIR depends on visual low-Level feature extraction these are based on color shades, texture, shape and spatial design. They have used LBP technique for texture and image analysis and furthermore it is contrasted and normal RGB color image descriptor technique. And afterward a correlative feature extraction strategy applying on RGB and LBP texture method has been proposed for CBIR.

Aditi Giri et. al.[9]focuses on color and texture based procedures for accomplishing productive and effective retrieval of images color feature extraction is finished by color histogram and color moment. Texture feature extraction is gained by wavelet [10] and gabor transform. For order of removed elements they have used SVM Euclidian separations are figured of each elements for likeness measures [3].

Shu Wang et.al. [11]Proposed an orderly method to reduce appearance SBIR gap. In this paper, outlines and extracted boundary are treated as set of contour segments, establishing the system for better draw edge description and noise affect reduction from another point. Entity edge selection algorithm is used to reduce the effect of noisy edges. In this paper, spatial constraints and coherent constraint are proposed to filter the false matches that reduce retrieval rate.

Swati Agarwal, et. al. [12]They proposed algorithm is illuminated for image retrieval dependent on shape and texture features not just based on color data. Firstly the input image is decayed into wavelet coefficients these wavelet coefficients give commonly even, vertical and inclining features in the image. Ensuing to wavelet transform (WT) and Edge Histogram Descriptor (EHD) is then used on favored wavelet coefficients to accumulate the data of first edge introductions. The gathering of DWT and EHD techniques expands the execution of image retrieval system for shape and texture based retrieval.

Min $\mathrm{H}$ et al. [13]present multi-feature combination technique which joins the color moment in RGB color space and color histogram in HSV color space for color feature extraction. The shape based features are extracted using improved Zernike moments and the texture feature extraction is done using GLCM. It combines these three features to describe an image. At last, the trial results are demonstrated using color, shape and texture features. The outcomes demonstrate that the image retrieval technique dependent on multi-feature combination has better retrieval performance.

\section{PROPOSED METHODOLOGY}

In this section, content based image matching systems has studied and reviewed, state of the current technology summarized in the previous chapters, results of which have guided us to design a CBIR system for future use. A through survey has revealed that various methods and combination of these methods can be applied on image for retrieval in both controlled and uncontrolled situations. Problem arises in uncontrolled condition when there are high rate of noises, different resolutions of the image etc. To mitigate the problems, the proposed an approach where the first method is based on registering the input images prior to their classification in the form of classes each class have multiple images and stored in database, after that apply retrieving process. This problem analytically depends on the strength and scalability of the content features, which used the characteristics of features vector. Also the paper examined an algorithm for retrieving analogous images which is based on color, texture and fusion for both local and global feature. 


\subsection{Proposed Algorithm}

Purpose: The algorithm is to retrieve images analogous to the query image.

$\mathrm{X}$ : database of images.

Input: Select a query image

Output: Similar to the input image get $\mathrm{n}$ images; $\mathrm{Q}^{+}, \mathrm{Q}^{-}$: relevant and non-relevant images, respectively.

Step 1: Input an image I with different three RGB color space of size $\mathrm{M} * \mathrm{~N}$

Step 2: Preprocessing to extract global and local features according to requirement.

Step 3: Color feature was extracted by computing color histogram and color structure descriptor for each of the color plane image in RGB, HSV color space.

Step 4: Apply texture descriptor EHD to extract global feature extraction from the image.

Step 5: Compute binary maps for each pixel for the given image apply bit plane slicing to compress the global information.

Step 6: Feature vector index was formed with no of elements, these stored as feature vector for image retrieval purposes as similarity measure.

Step 7: Compare query image feature vector with database image feature vector and matching performed by different fusion methods and get different results according to selection.

Step 8: Store all matching images in the result section and also display them.

Step 9: If similar results are not attained then repeat the steps from 2 to 8 for the entire image present in database.

Step 10: Retrieve the images for another query, repeat the process if required then exit.

\subsection{Block Diagram}

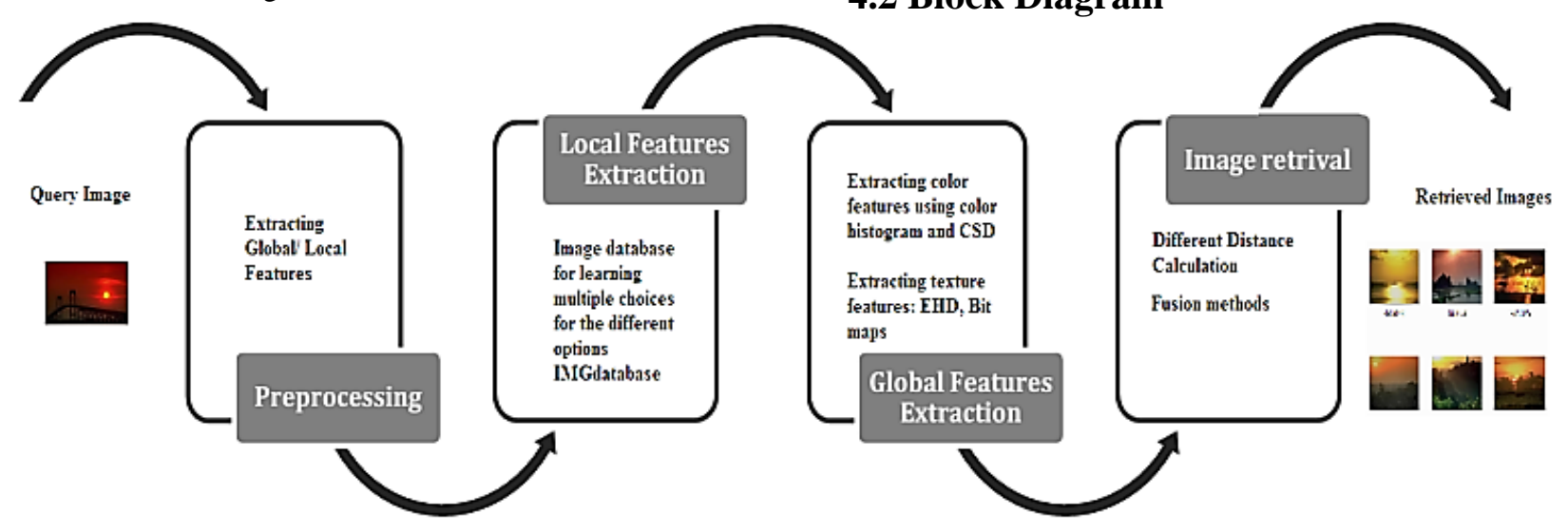

Fig 1: Block Diagram of proposed approach

\subsection{Query Image}

First select an image that will go through image retrieval process. Whose main aim is to obtain similar images? In this study IMG database containing color images was used.

\subsection{Preprocessing}

In this phase an image processing feature extraction type was selected and apply preprocessing according the requirement. The image data is highly non-trivial.

\subsection{Feature Extraction}

There has been tremendous work on various ways to deal with the recognition of different kinds of features in images. These features can be classified as follows:

\subsubsection{General features:}

Features in this category are all application independent, e.g. color, texture, and shape. According to concept level, they can be further divided into:

- Local features: The local image description is established on the reason that images can be described by characteristics registered on regions of the image. Can be determined over the consequences of image division and edge detection algorithms. Object shape is an example of such feature [14].

- Global features: The global image descriptor is composed by color and texture features being computed
- Pixel-level features: Can be determined at every pixel, for example color, area, and the first and second derivatives of gray-scale values at every pixel

Pixels are extracted and stored in an array. The array contains the RGB components of each pixel. Each pixel in the image is then processed to identify the feature vectors of the image. Edges were used as the only feature vector

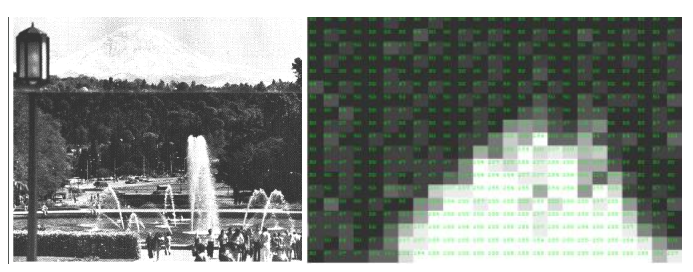

Fig 2. Digital Representation of an image

on the whole image. Typically, they are in truth the measurable properties of an image, e.g. histogram, mean, variance, and moment [14].

Feature Extraction is done by using colors and textures. For color feature extraction, color histograms and color moment and color sketch are used. For texture feature extraction, Edge histogram descriptor is used.

- The images are recorded with their equivalent features 
such as color, texture.

- These extracted features will be forwarded to Feature Vector Module.

\subsection{Color Features Extraction}

Color is one of the dominant and recognizable visual features used in CBIR, primarily as a result of the effortlessness of separating color data from the images. It prompts minimum error as it is generally robust to foundation complication, viewing point, Translation and free of image size and introduction. Color- based image retrieval provides an intelligent and automatic solution; a better performance can be obtained by using a combination of multiple features for image retrieval which indirectly results in a complex algorithm with increased memory space for storing feature vectors and increased query execution time.

\subsection{Color Space selection and color quantization}

A digital image in this context is set of pixels and each pixel has intensity and color, Colors can be represented using different color models depending in the application such as RGB, HSV etc.

\subsection{Color Descriptor}

\subsubsection{Color histogram}

The RGB color space for the histograms was used. Just minor differences have been seen with other color spaces for the histogram. Color Histogram $\mathrm{H}(\mathrm{M})$ is an inaccessible likelihood function of the image color [2]. The color histogram is a standard show of color trademark in CBIR. A color histogram for a particular image is represented by a vector:

$$
H=\{H[0], H[1], H[2], H[3], \ldots \ldots \ldots H[i], \ldots \ldots \ldots, H[n]\}
$$

In this equation i represents color bin in the color histogram and $\mathrm{H}[\mathrm{i}]$ represents the number of pixels of color $\mathrm{i}$ in the image, $\mathrm{n}$ is the total number of bins used in color histogram. Each pixel in an image will be assigned to a bin of a color histogram.

This likelihood work is used for the assurance of joint likelihood work for the forces of the three color channels. Further casually, the normalized color histogram $\mathrm{H}^{\prime}$ is characterized as.

$$
=\left\{\begin{array}{c}
\mathrm{H}^{\prime} \\
\left.\mathrm{H}^{\prime[0]}, \mathrm{H}^{\prime[1]}, \mathrm{H}^{\prime[2]}, \mathrm{H}^{\prime[3]}, \ldots \ldots \ldots \mathrm{H}^{\prime[\mathrm{i}]}, \ldots \ldots \ldots, \mathrm{H}^{\prime}[\mathrm{n}]\right\}
\end{array}\right.
$$

WhereH' $=\frac{H[i]}{P}$, is the total number of pixels of an image

\subsubsection{Color Structure Descriptor (CSD)}

The CSD uses the HMMD color space and an $m \times m$ structuring element to represent local color organization in an image by counting the amount of times a particular color appears within the structuring element while the latter scans the image. Let $C 0, C 1, \ldots, C K-1$ denote the $K$ quantized colors. Color histograms in each bin $\mathrm{k}$ the quantity of organizing components in the image containing at least one pixel with color $\mathrm{Ck}$. The bin estimations of the CSD are standardized by the number of locations of the organizing component and lie in the range $[0.0,1.0]$. The standardized qualities are then non-linearly quantized to 8 bits per bin.

\subsubsection{Color grid}

In this, segments each image to $m \times n$ grid each cell of which will be analyzed separately. In this user fill each grid by different color and blobs on the query area because images were divided into grids, and the color and texture features were determine in these grids. A normal Color Histogram is applied on each sub-image created by dividing the original image [2]. The most common color of each cell will constitute a part of a 1 by $\mathrm{m}$ dimensional feature vector [11] [10].

\subsection{Texture Feature Extraction}

The second element of the new system is the texture feature. For this purpose, EHD algorithm is used. Texture is an important feature of expected images. A variety of techniques have been proposed for estimating texture comparability. These strategies ascertain proportions of image texture, for example, the level of differentiation, coarseness, directionality and consistency [8]; or periodicity, directionality and randomness.

\subsection{Edge Histogram Descriptor}

The edge histogram descriptor represents to the spatial dispersion of five kinds of edges, to be specific four directional edges and non-directional edges. The edge histogram descriptor takes after the color layout descriptor (CLD) in its standard of capturing the spatial conveyance of edges which is valuable in image coordinating regardless of whether the texture itself isn't homogeneous [15].

\subsection{Fusion}

Combination at the feature level includes the mix of feature sets to different modalities. Extracts different features, for example edges, corners, lines, texture parameters and so forth., from various information sources and afterward joins them into at least one element maps that might be used rather than the first information for further preparing.

\subsection{Similarity Measures}

Broadly used classes of image comparability measurements include playing out some activity on the differences between relating pixels in two images, then summing over these modified differences.

To find the similarities between query image and the images in the database distance between two images is used. The proposed technique used the Euclidean separation between the two element vectors of the images. The separation can be determined by:

\subsection{Euclidean Distance}

Euclidean distance calculates the separation between query image and database image. Query image are compared with database images. The Euclidian Distance is general the shortest distance between two points.

$$
E D\left(A^{I}, A^{Q}\right)=\sqrt{\sum_{n=1}^{n=m}\left(A_{n}{ }^{I}-A_{n}{ }^{Q}\right)^{2}}
$$

Where $A^{I}$ is the stored images in the database

$A^{Q}$ is the Query image for the retrieval

The maximum size of the distance cannot be specified before. There normalization is necessary too.In this the Quadratic distances are calculated between the two pixels the matching images is made.

\subsection{Quadratic Distance}

The distance treats all bins of the histogram completely autonomously and does not represent the way that specific sets of bins compare to features which are perceptually more 
comparative than different sets [16]. To solve this problem, quadratic form distance was used

$$
D Y 1(I, J)=\sqrt{\left(H_{I}-H_{J}\right)^{T} * A *\left(H_{I}-H_{J}\right)}
$$

Where $\mathrm{H}_{\mathrm{I}}$ and $\mathrm{H}_{\mathrm{J}}$ are vectors that list all the entries in $h_{i}(I)$ and $h_{j}(J)$ anda $_{i j}$ denote the similarity between bin $\mathrm{i}$ and j.

\section{IMPLEMENTATION RESULTS}

\subsection{Dataset}

The Dataset contains around 2000 images in JPG format there are images of animals, sceneries, people, nature, etc shown in figure 3. The set of images used in the proposed system consist of 2000 images.

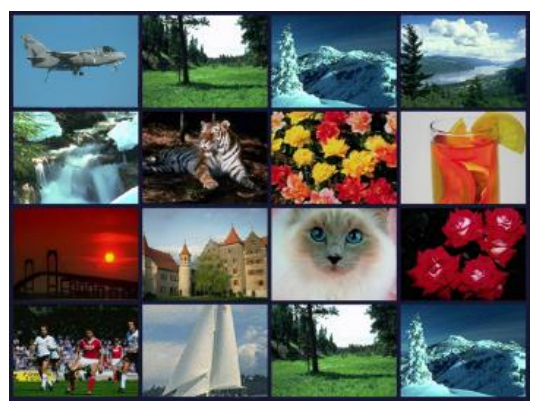

Fig 3: Sample images from IMG database

\subsection{Experimental Result}

Here, our proposed approach was tested and the results are depicted. Retrieval adequacy of the proposed system, was checked by choosing a few images randomly and retrieving some relevant images.

\subsubsection{Graphical user interface}

The GUI plays an important role in the browsing and retrieval processes as through it, these processes are initiated and their results are presented. For the sake of easiness and debugging our GUI was designed as a MATLAB GUI.

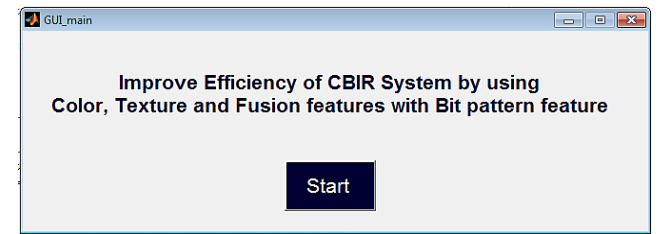

Fig 4: Main GUI environment

A CBIR system should offer easy and optimized ways first to select the query image, second options to select the features, distance measure and fusion levels for retrieval, third to a user can use the slide bar to change the weight of a given feature, forth to pick the retrieval option, and finally it should offer a way to visualize the results in an interpretable way.

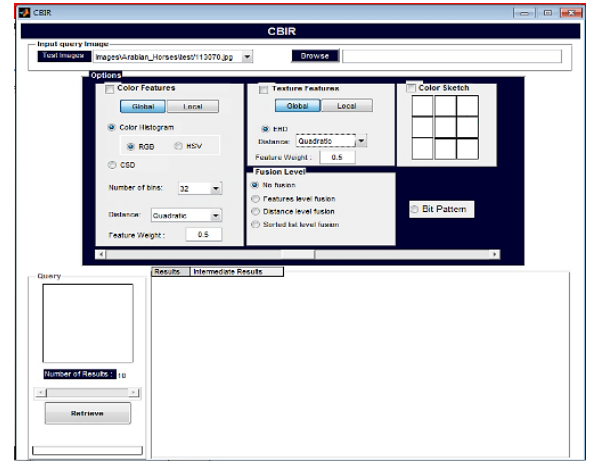

Fig 5.GUI environment after clicking on start

\subsubsection{Query by content}

The user can input a query by an image from the database or external image.

\subsubsection{Query Specification}

Specifying what kind of images a user wishes to retrieve from the database can be done in many ways[17]. Commonly used query formations are: category browsing, Category browsing is to browse through the database according to the category of the image.

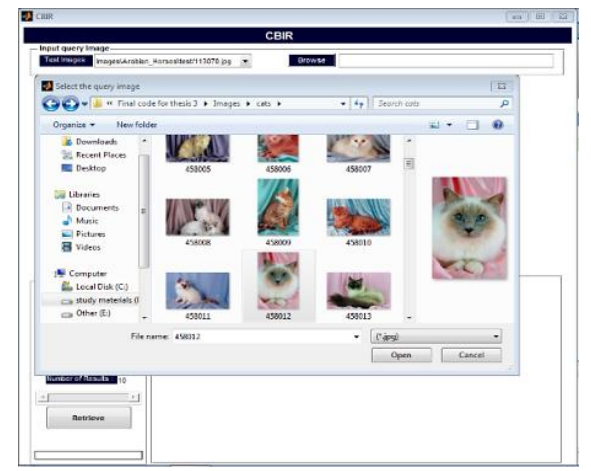

Fig 6: Interface to select input query image

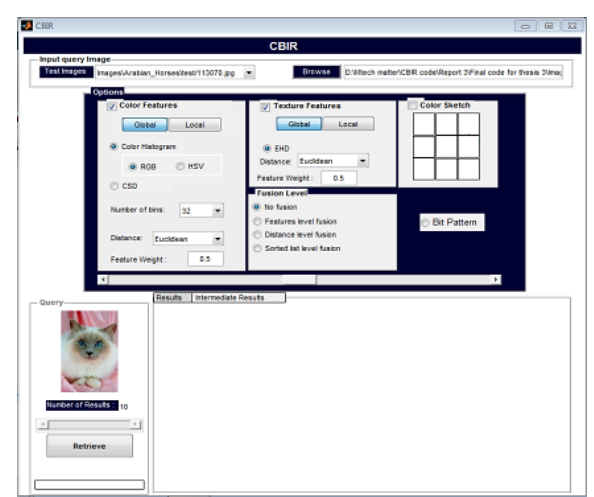

Fig 7: GUI after selecting input query image

\subsubsection{System Evaluation and displaying most relevant images}

The differences between the distances are used to find the 10 most relevant images from the dataset. These images are retrieved from the dataset and displayed in the GUI Results environment window.

The first test is selecting a random image from the building class. Submit the image to the system and retrieve the top 20 images that are similar to the query image. As can be seen from figure 8 . 


\subsubsection{Different options apply on images}

1. Global: Color Features (Color Histogram, 32, RGB) + Texture Features (EHD) + Feature level fusion + Bit Pattern + Quadratic distance

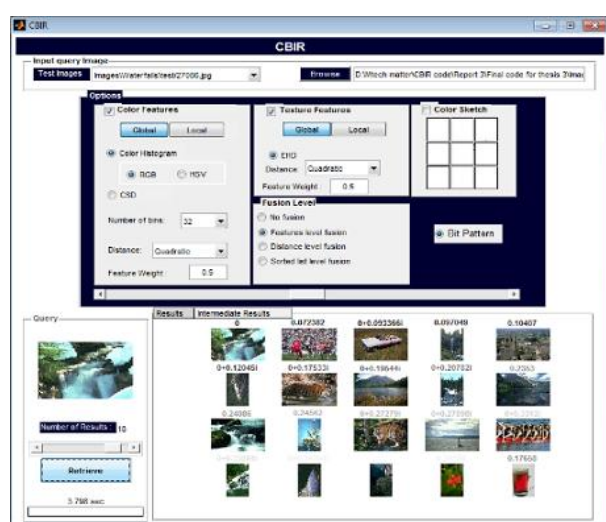

Fig 8: Retrieval results for waterfall image (Feature level)

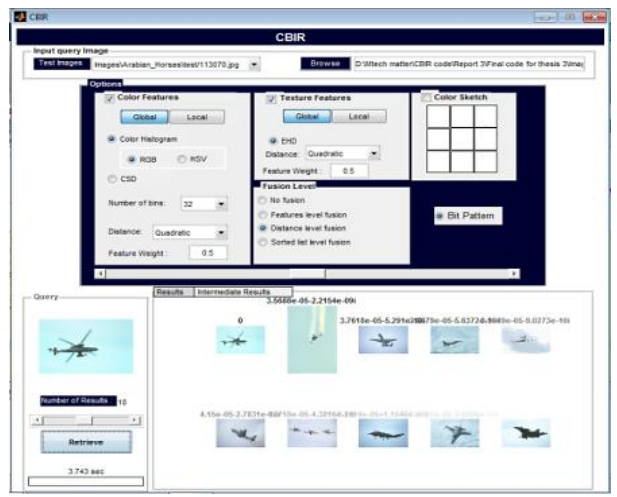

Fig 9: Retrieval results for Aviation Photography image (Distance level)

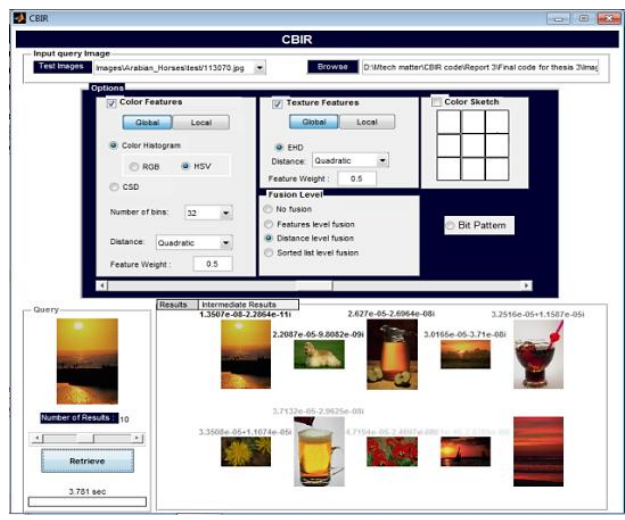

Fig 10: Retrieval results for drink image (HSV + Distance level)

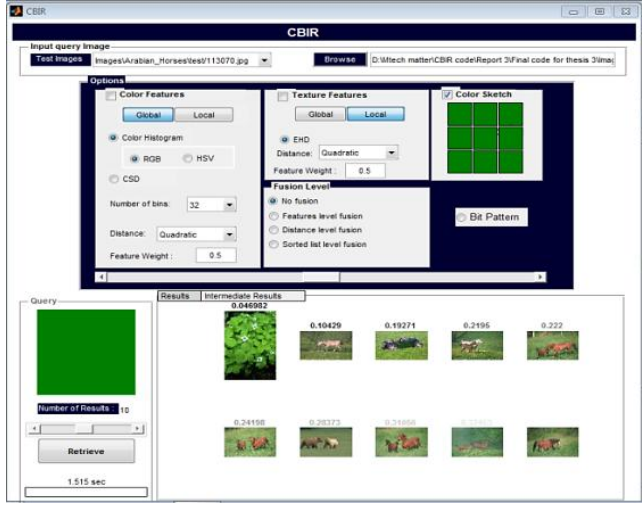

Fig 11: Results based on Color grid

\subsubsection{Performance Evaluation matrices}

Generally, calculate the two performance evaluation metrics that are Precision and Recall. Precision measures the accuracy of the retrieval and Recall measures the robustness. The metrics are defined as:

$$
\begin{gathered}
\text { Precision }=\frac{\text { Number of relevent images retrieved }}{\text { Total number of images retrieved }} \\
\text { Recall }=\frac{\text { Number of relevant images retrieved }}{\text { Total number of relevent images in the dataset }}
\end{gathered}
$$

\subsubsection{Accuracy}

An accuracy of the system can be defined as the half the fraction sum of precision and recall values, which can be expressed as,

$$
\text { Accuracy }=\frac{\text { Precision }+ \text { Recall }}{2}
$$

Finally, compare our planned system with other existing systems and show the efficiency of the proposed system. To test the system, 200 different images was selected from database and from different classes (10 images from each class).

Table 2. Accuracy of images retrieved

\begin{tabular}{|c|c|c|}
\hline Query images & Accuracy & Time(Sec) \\
\hline Query 1 & $94.44 \%$ & 3.753 \\
\hline Query 2 & $95 \%$ & 3.753 \\
\hline Query 3 & $90 \%$ & 3.781 \\
\hline Query 4 & $100 \%$ & 3.893 \\
\hline Query 5 & $100 \%$ & 1.515 \\
\hline Average & $\mathbf{9 5 . 8 0 \%}$ & $\mathbf{3 . 3 3 9}$ \\
\hline
\end{tabular}




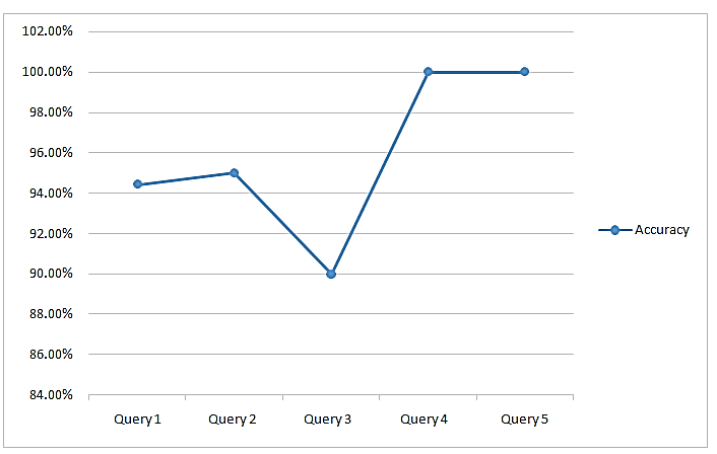

Fig 12: Comparative analysis of accuracy

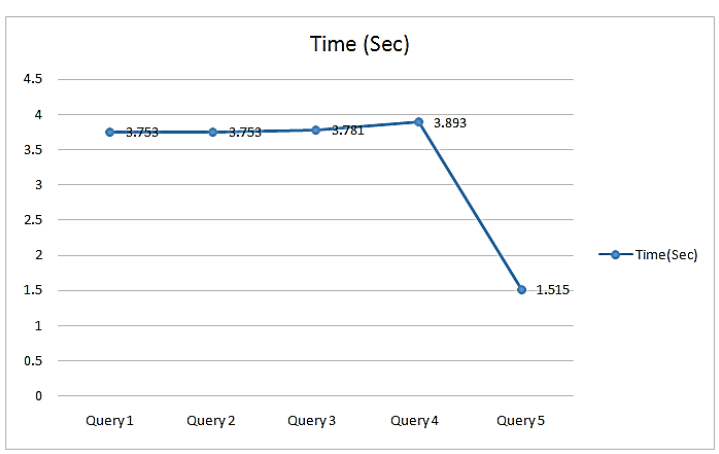

Fig 13: Comparative analysis of time

Table 3. Accuracy and Time comparison of the proposed approach

\begin{tabular}{|c|c|c|c|c|c|c|}
\hline \multirow{2}{*}{ Category } & \multicolumn{2}{|c|}{ Color Based } & \multicolumn{2}{c|}{ Texture Based } & \multicolumn{2}{c|}{$\begin{array}{c}\text { Bit Pattern } \\
\text { Based }\end{array}$} \\
\cline { 2 - 7 } & $\begin{array}{c}\text { Acc } \\
\text { urac } \\
\mathbf{y}\end{array}$ & $\begin{array}{c}\text { Time } \\
\text { (Sec) }\end{array}$ & $\begin{array}{c}\text { Accurac } \\
\mathbf{y}\end{array}$ & $\begin{array}{c}\text { Time } \\
(\text { Sec) }\end{array}$ & $\begin{array}{c}\text { Accur } \\
\text { acy }\end{array}$ & $\begin{array}{c}\text { Time } \\
\text { (Sec) }\end{array}$ \\
\hline Horses & $88 \%$ & 1.841 & $84 \%$ & 3.431 & $93 \%$ & 1.231 \\
\hline Castles & $87 \%$ & 1.487 & $81 \%$ & 3.482 & $89 \%$ & 1.367 \\
\hline $\begin{array}{c}\text { Aviatio, } \\
\text { Photography }\end{array}$ & $85 \%$ & 1.743 & $80 \%$ & 3.483 & $87 \%$ & 1.148 \\
\hline Drinks & $83 \%$ & 1.457 & $67 \%$ & 3.528 & $90 \%$ & 1.237 \\
\hline Tulips & $88 \%$ & 1.475 & $75 \%$ & 3.720 & $91 \%$ & 1.394 \\
\hline Average & $\mathbf{8 6 . 2}$ & $\mathbf{1 . 6 0 1}$ & $\mathbf{7 7 . 4 \%}$ & $\mathbf{3 . 8 2 9}$ & $\mathbf{9 0 \%}$ & $\mathbf{1 . 2 7 5}$ \\
& $\%$ & & & & & \\
\hline
\end{tabular}

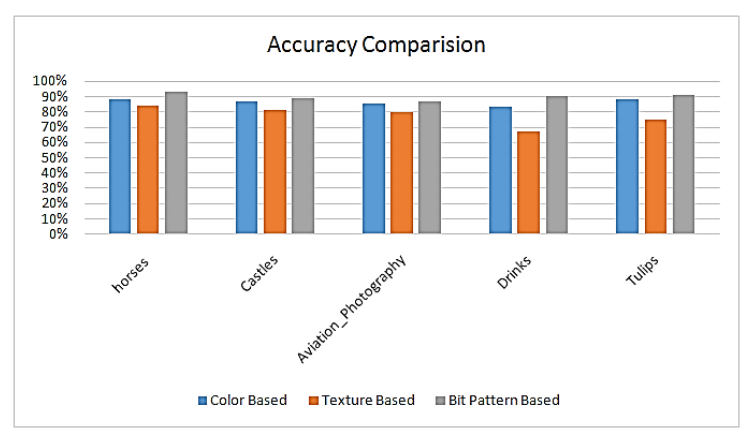

Fig 14: Accuracy comparison with different options for different categories

\section{CONCLUSION AND FUTURE WORK}

This system works for searching and retrieving images. Regarding the "huge" size of the database, our system provided good results. Using more performance measures fine adjustments can be made with more features and possibly provide the users with the best options of retrieval as default parameters, the system attempts to present a hybrid technique for CBIR, which uses the combination of Feature extraction with better image retrieval accuracy. The proposed system matches the images if the dominant color is similar. This limitation can be resolved by using more than one feature options to represent the image. In the next section, some ideas to enhance the system have been stated.

The present work can be extended by improving the recognition rate by increasing the feature vectors and using a combined approach to retrieve similar images. The present implementation has an application in lot of fields such as military, medicine, crime detection, etc. An embedding of number plate recognition program with this method will help to identify vehicles automatically, which will help in finding stolen vehicles. Given an image database of vehicles, the program can retrieve similar images of cars from database in accordance to input image. Furthermore using number plate recognition program the user can search for number plates by giving the number as query, and retrieve information about the vehicle. Further studies regarding measuring the performance of more options and $3 \mathrm{D}$ visualization of the search results are currently being investigated.

\section{REFERENCES}

[1] Memon, M., H., Li,J., Memon, I., Shaikh, R., A., and Mangi, F., A. 2015. Efficient object identification and multiple regions of interest using cbir based on relative locations and matching regions. International Computer Conference on Wavelet Active Media Technology and Information Processing

[2] Poursistani, P., Nezamabadi-pour H., Moghadam, R., A., and Saeed, M. 2011. Image indexing and retrieval in JPEG compressed domain based on vector quantization. Math. and Comp. Modeling (March 2013), 1005-1017

[3] P. Poursistani, H. Nezamabadi-pour, R. A. Moghadam, and M. Saeed, M. 2011. Image indexing and retrieval in JPEG compressed domain based on vector quantization. Math. and Comp. Modeling (March 2011), 1005-1017.

[4] Sugamya, K., Pabboju, S., Babu, A.V. 2016. A Cbir Classification Using Support Vector Machines. 2016 International Conference on Advances in Human Machine Interaction, (March 2016), 1-6

[5] Ashraf R., Bashir K., Mahmood T. 2016. Content-based image retrieval by exploring bandletized regions through support vector machines. J Inf Sci Eng. 32(March 2016), 245-269.

[6] Pushpalatha S., Nikkam, D., Nagaratna, P., Hegde and B. Eswar Reddy, 2015. Decomposition-Based Shape Template Matching for CBIR System, International Conference on Computational Intelligence and Computing Research

[7] Gupta E. and Kushwah R., S. Combination of Global and Local Features using DWT with SVM for CBIR. 2015 4th International Conference on Reliability, Infocom Technologies and Optimization (ICRITO) (Trends and Future Directions), 
[8] Kumar K., Li, J-P., and Abidin Z., Complementary Feature Extraction Approach In CBIR, 2015 12th International Computer Conference on Wavelet Active Media Technology and Information Processing (ICCWAMTIP)

[9] Giri,A., and Meena, Y. K 2014. Content based image retrieval using integration of color and texture features. Int. J. Adv. Res. Comp. Eng. Tech. (April 2014), 14511454.

[10] Hazra., D 2011. Retrieval of color image using color correlogram and wavelet filters. Proceedings of International Conferefence on Advances in Computer Engineering.

[11] Shu Wang, S., Zhang, J., Han,, T., X., and Miao, Z. 2015. Sketch-Based Image Retrieval Through Hypothesis-Driven Object Boundary Selection With HLR Descriptor. IEEE Transactions on Multimedia 17 (July 2015), 1045-1057.

[12] Swati Agarwal, S., Verma, A. K. Singh, P. 2013. Content Based Image Retrieval using Discrete Wavelet Transform and Edge Histogram Descriptor. International Conference on Information Systems and Computer
Networks, proceeding of IEEE xplore-2013.

[13] Min Huang, M., Shu, H., Mab, Y., and Gong,Q. 2015. Content-based Image Retrieval Technology using MultiFeature Fusion. Optik-International Journal for Light and Electron Optics 126 (October 2015) 144-2148.

[14] Gbèhounou, S., Lecellier, F., Maloigne C. 2016. Evaluation of local and global descriptors for emotional impact recognition. J.Vis. Comm. Image Rep. (March 2016), 8.

[15] Premchaiswadi, W., and Tungkasthan, A. A Compact Auto Color Correlation using Binary Coding Stream for Image Retrieval. Proceedings of the 15th World Scientific and Engineering Academy and Society (WSEAS) International conference on Computer, Recent Researches in Computer Science.

[16] Wan, X., and Kuo, C.C., 1996. Image retrieval with multiresolution color space quantization. Proccedings SPIE 2898, Electronic Imaging and Multimedia Systems.

[17] Silakari, S., Motwani, M and Maheshwari, M. 2009. Color image clustering using block truncation algorithm.Int. Jour. of Comp. Science. (Sep 2009), 3135 